\title{
Considerations and recommendations on traditional and non-traditional uses of excipients in oral drug products
}

\author{
Panayiotis P. Constantinides ${ }^{1 *}$, Subhashis Chakraborty ${ }^{2}$ and Dali Shukla ${ }^{3}$
}

\begin{abstract}
Excipients represent diverse classes of molecules, small molecules or macromolecules, with versatile structures within a given class and are from natural, semi-synthetic and synthetic sources. They are essential ingredients in drug products independently of the route of administration where both traditional and non-traditional uses are present. Beyond their traditional use as formulation and manufacturing aids, certain excipients exhibit biological effects and thus can be used either as atypical active pharmaceutical ingredients alone or synergistically with conventional active pharmaceutical ingredients to affect the overall pharmacokinetics/ pharmacodynamics and therapeutic effect(s) of the co-administered drug(s). In reference to oral drugs, biological effects of the excipients that contribute to improved drug absorption and pharmacokinetics include their ability to modulate drug dissolution, intestinal membrane permeability, gut wall metabolism and efflux pumps. These non-traditional uses of excipients are illustrated in representative case studies from published literature. From a drug development and regulatory perspective, it is apparent that an appropriate excipient classification system is considered which is applied to new uses of existing excipients as well as to the development of novel excipients and segregates biologically active excipients from the larger pool of excipients. This classification system should be a useful tool to pharmaceutical scientists and its successful implementation and broader acceptance requires input and ownership from all major stakeholders, that is, the excipient manufacturers, the end users, excipient forums and discussion groups such as the International Pharmaceutical Excipients Council (IPEC) and regulatory agencies.
\end{abstract}

Keywords: Excipients, Traditional and non-traditional uses, Oral drug products, Biologically active excipients, Drug transport, Permeability, Metabolism, Pharmacodynamics, Pharmacokinetics, Atypical active pharmaceutical ingredient, Excipient classification system

\section{Background}

In order to harvest the maximum therapeutic activity of the drug molecules, appropriate dosage forms are designed using various additives called 'Excipients' (Rowe et al. 2009). It is due to the use of excipients that the drug can be delivered to the patients in a presentable form that meets the targeted dose requirements. Excipients represent diverse classes with versatile structures and are of natural or semi-synthetic origin, small molecules and macromolecules and represent all states of matter, that is, gases, liquids, semi-solid and solids. Examples of small

\footnotetext{
* Correspondence: constantinpp@aol.com

'Biopharmaceutical \& Drug Delivery Consulting, LLC, P. O. Box 7565, Gurnee, IL 60031-6226, USA

Full list of author information is available at the end of the article
}

molecule excipients include, mannitol and dextrose, sodium chloride, vitamins such as ascorbic acid (vitamin C) and $\alpha$-tocopherol (vitamin E), calcium carbonate and calcium phosphates (Rowe et al. 2009), whereas macromolecular excipients include, polyethylene glycols, methacrylates, povidone, aluminosilicates and hydrocolloids, such as starches, cellulose esters, gums, gelatin, alginates, carrageenan and pectin (Rowe et al. 2009). Excipients are manufactured in large quantities often through continuous processing in dedicated plants and are available in several grades. Traditionally excipients are regarded as "inert carriers" (inactives). However, they may also exert biological activity as modulators of drug absorption and metabolism or as atypical active pharmaceutical ingredients. 
Many excipients used to solubilize poorly soluble drugs and are present in marketed oral and parenteral drug products have been reported in the literature (Strickley 2004; Wasan 2007). Alteration of the permeability of the drug, however, requires chemical modification of the drug molecule which often alters its therapeutic activity and is, therefore, impractical. Non-structural approaches to improve mucosal membrane permeability rely on the use of permeation enhancers (Aungst 2012), such as lipidic and polymeric excipients. The toxicity profile and safety of the particular enhancer need to be rigorously established by also considering the fact that excipients levels in oral dosage forms are significantly higher compared to the levels of the active pharmaceutical ingredients (Whitehead et al. 2008; Maher et al. 2009). Examples of permeation enhancers, a use of excipients that is considered nontraditional, are listed in Table 1 (Aungst 2012; Whitehead et al. 2008; Maher et al. 2009).

The appropriate selection and use of excipients to deliver drugs is dependent on the requirements during fabrication, desired dissolution and release profile, organoleptic characteristics of the formulation, drug stability and overall physiochemical characteristics. Analyzing the above selection criteria clearly indicates that some excipients used with an objective to modify the in-vivo dissolution and release profile of the drugs can cause significant alteration in its pharmacokinetic profile, resulting in alteration in its pharmacodynamic activity. Furthermore, as indicated in Table 2, certain excipients exhibit biological activity by inhibiting metabolic enzymes in the gut wall and intestinal efflux pumps (Constantinides et al. 2007; Chen 2008). If used intentionally, such excipients by virtue of their ability to increase the drug's solubilization capacity in the intestine, metabolic stability and membrane transport may prove to be extremely useful to improve bioavailability; otherwise it may result in severe

Table 1 Intestinal Permeability Enhancers (Aungst 2012; Whitehead et al. 2008; Maher et al. 2009)

\begin{tabular}{ll}
\hline Chemical Class & Molecule \\
\hline Bile Salts & Sodium cholate/deoxycholate \\
Non-ionic surfactants & Cremophor EL/RH40, Solutol-HS-15, TPGS, \\
Tween 80 & Phospholipids (lecithin) \\
Ionic surfactants & Stearylamine, cetyl phosphate \\
& Lauroyl- and palmitoyl-carnitine chloride \\
& Sodium lauryl sulfate \\
& Dioctyl sodium sulfosuccinate \\
Fatty acids/salts & Oleic Acid, sodium caprylate, sodium caprate \\
Glycerides (C8/10) & Capmul MCM, Labrasol \\
Polymers & Chitosan, polycarbophil, starch, carrageenan \\
Other & Sodium salicylate, N-methylpiperazine \\
\hline
\end{tabular}

Table 2 Examples of Excipients Inhibiting In Vitro/In Vivo Metabolic Enzymes and Membrane Transporters (Chen 2008; Constantinides et al. 2007)

\begin{tabular}{lll}
\hline Excipient & CYP3A & P-gp \\
\hline C8-C20 fatty acid glycerol and PEG esters: & - & + \\
Cremophor EL and RH40, Solutol HS-15, & & \\
Labrasol, Softigen 767, Aconnon E & & \\
Sodium Caprate & - & + \\
Polysorbates : Tween 80, Tween 20 & + & + \\
Sucrose esters : sucrose monolaurate & - & + \\
Tocol esters : TPGS & - & + \\
PEG 300/400 & - & + \\
Triton X-100 & - & + \\
Pluronic block copolymer (Pluronic F68) & + & + \\
Amphiphilic diblock copolymers (Me-PEG-b-PCL) & - & + \\
Peppermint Oil & + & - \\
\hline
\end{tabular}

toxic plasma drug concentration leading to unwanted side effects. A realization in recent years is also the fact that some excipients are also used as atypical active pharmaceutical ingredients alone or synergistically with other active pharmaceutical ingredients (Drakulich 2011; Boddu et al. 2015). Atypical active pharmaceutical ingredient is defined as an excipient that is being used as the active pharmaceutical ingredient in the formulation. Over- thecounter (OTC) products are examples where one or more excipients are used as atypical active pharmaceutical ingredients in oral, topical, parenteral and ocular dosage forms (Boddu et al. 2015). The term atypical active pharmaceutical ingredient is adapted in the present commentary except in the cited references where the synonymous short version "atypical active" appears.

\section{Case studies}

Although the presently available list of excipients in the market is unending, the excipients which have shown to significantly improve the therapeutic efficacy of drugs are either small molecules or macromolecules and include lipids, surfactants, oils, cyclodextrins, ion exchange resins, hydrogels, hydrophobic polymers and other (Strickley 2004; Wasan 2007; Constantinides et al. 2007; Chen 2008). In the case of lipid excipients for example, some of their traditional uses include, drug solvents and stabilizers, surfactants, antioxidants and vaccine adjuvants. Non-traditional uses of lipid excipients include membrane permeability modulators (Table 1 ), gut wall metabolism and efflux pump inhibitors (Table 2) as well as atypical active pharmaceutical ingredients (Constantinides et al. 2007; Chen 2008); Boddu et al. 2015; Constantinides et al. 2006; Shukla et al. 2011; Chakraborty et al.; 2009). These traditional and non-traditional functions of lipid excipients are illustrated in Tables 1 and 2 and in Fig. 1. Polyionic hydrocolloids, such as alginate and chitosan, 


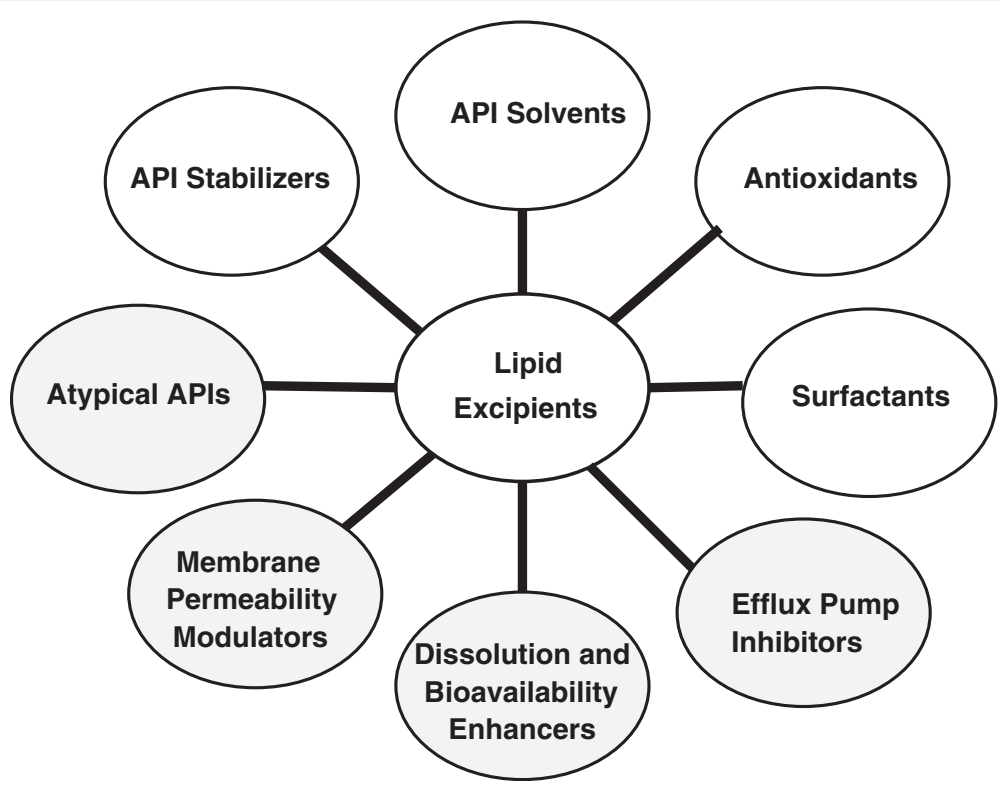

Fig. 1 Traditional and Non-Traditional Uses of Lipid Excipients. Non-traditional uses are highlighted

commonly used as gelling agents, have also been used non-traditionally for the intestinal delivery of peptide and protein drugs (George et al. 2006). Likewise, thickening agents such as, layered 2:1 phyllosilicates (clay minerals) have been evaluated for non-traditional uses, as antimicrobial agents for example (Haydel et al. 2008). Another example of a non-traditional use, include surface modified nanostructured aluminosilicates (montmorillonite) which have recently been employed to inhibit the absorption of dietary cholesterol in animals (Gershkovich et al. 2009). Diverse applications of tocol family of compounds (tocopherols and tocotrienols) and tocol esters, such as tocopherol succinate and $\alpha$-tocopherolpolyethylene glycol 1000-succinate (TPGS) include their use as drug delivery vehicles, antioxidants and therapeutic agents (Constantinides et al. 2006). Increased membrane permeability of low permeable substances has been successfully demonstrated in the presence of basic butylated methacrylic polymers (Alasino et al. 2005; Grube et al. 2008). Various methacrylic polymers marketed as Eudragit ${ }^{\circ}$, beyond their traditional use as enteric coating and controlled release excipients, have also been investigated as functional excipients in solid dispersions of poorly soluble drugs to enhance drug solubilization and physical stability (Six et al. 2004; Qi et al. 2008). The latter use of these materials is regarded as non-traditional. The above examples of excipients can be distinctly segregated from other excipients like fillers, binders, lubricants, disintegrants, glidants, colors, flavors, preservatives, antioxidants, plasticizers etc. (Rowe et al. 2009), which are used as manufacturing aids and have insignificant relation with the in-vivo performance of the drug. Table 3 illustrates traditional and non-traditional uses of pharmaceutical excipients as applied to minerals (Rowe et al. 2009; Boddu et al. 2015; Gershkovich et al. 2009, Constantinides 2014). Detailed information on non-traditional uses of the various pharmaceutical minerals listed in Table 3 including their use as atypical pharmaceutical ingredients in OTC medications has been reported elsewhere (Boddu et al. 2015; Gershkovich et al. 2009, Constantinides 2014). There are indeed multiple uses of a particular class of excipients, such as minerals, a characteristic that also applies to other classes of excipients.

Table 3 Traditional and Non-Traditional Uses of Minerals in Pharmaceuticals (Rowe et al. 2009; Boddu et al. 2015; Haydel et al. 2008, Gershkovich et al.; 2009; Constantinides 2014)

\begin{tabular}{|c|c|c|}
\hline Minerals & Traditional Uses as Functional Excipients & $\begin{array}{l}\text { Non-Traditional Uses as Atypical Active } \\
\text { Pharmaceutical Ingredients }\end{array}$ \\
\hline $\begin{array}{l}\text { Kaolin, Montmorillonite (bentonite), Magnesium } \\
\text { Aluminum Silicate, Talc (hydrated magnesium } \\
\text { silicate), Precipitated Calcium Carbonate, } \\
\text { Calcium sulfate, Dibasic Calcium Phosphate, } \\
\text { Magnesium Carbonate, Colloidal Silicon } \\
\text { Dioxide, Sodium Chloride }\end{array}$ & $\begin{array}{l}\text { suspensions, gels, lotions, magmas and } \\
\text { milks, adsorbents, fillers and diluents } \\
\text { disintegrants, lubricants anti-adherents, } \\
\text { glidants }\end{array}$ & $\begin{array}{l}\text { gastric antacids, laxatives, anti-diarrhea agents, } \\
\text { topical OTC medications, antimicrobial agents: } \\
\text { antiviral, antibacterial antifungal, inhibitors of } \\
\text { the absorption of dietary cholesterol }\end{array}$ \\
\hline
\end{tabular}


Non-traditional uses of excipients in oral drug products as well as in dietary supplements has been the subject of various conferences and workshops and more recently of a symposium at the 2014 AAPS Annual Meeting in San Diego with a theme Excipients as Atypical Actives in Nutraceuticals and Pharmaceuticals: Application and Development Considerations (Constantinides 2014). In this latter program talks included, dietary role of excipients, excipients as inhibitors of the absorption of dietary cholesterol, development challenges in the utilization of atypical actives in drug products and regulatory perspectives surrounding non-traditional uses of excipients.

In a regulatory note, Chen et al.; (Chen et al. 2013) reviewed and analyzed pharmacokinetic data on the use of osmotically active excipients, such as, sorbitol, mannitol, D-xylose and PEG400 on the bioavailability and bioequivalence of BCS III drugs that included, ranitidine, cimetidine and amoxicillin, and concluded that, "a better understanding of the dose-response relationship of an "active" excipient can facilitate its optimal use in formulations and further provide opportunities of biowaivers while ensuring product quality and performance". It was pointed out by the authors that "from a regulatory viewpoint, the importance of recognizing the presence of an "active" excipient in the formulation cannot be overemphasized during the development for both generic and innovator companies". They further suggested that additional mechanistic studies are needed in order to shed light into the effects of "active" excipients on drug absorption and bioavailability/bioequivalence (Chen et al. 2013). These findings are certainly relevant to the perspectives and recommendations presented in this commentary and regulatory agencies represent a key stakeholder on the qualification and classification of excipients as discussed below.

The effect of twenty commonly used excipients on the oral bioavailability of nalbuphine, a potent opioid analgesic, in rats and humans was investigated and reported (Wang et al. 2014). The excipients used included, surfactants (Tween 20/40/80, Cremophor EL/RH40, Solutol HS-15, Pluronic F68 and sodium lauryl sulfate), PEG 400 and PEG2000, as well as preservatives (methyl and propyl parabens, and sodium benzoate). Improvement in the oral bioavailability of nalbuphine was correlated to the ability of these excipients to inhibit the activity of UDP-glucoronosyltranferase 2B7 activity, the major metabolic enzyme for nalbuphine, with Tween 20 and PEG400 being the most active, alone and in combination (Wang et al. 2014).

It should also be pointed out that several biomaterials/ excipients used in various drug carriers such as microand nanoparticles and administered by the oral, parenteral and other routes of administration, are reported to exhibit bioactivities in the body by modulating cellular and physiological processes, well beyond their primary role to modify the pharmacokinetics and biodistribution of the drug (Yeo et al. 2015). These pharmaceutical excipients include, chitosan and chitosan derivatives, hyaluronic acid, pluronics, polar lipids such as pegylated phospholipids and cationic lipids, cyclodextrins and $\mathrm{N}-(2-$ hydroxylpropyl) methacrylamide copolymers (Yeo et al. 2015). Reported bioactivities of these materials which are either known or newly discovered include, suppression of pro-inflammatory cytokine production and slight increase in tumor burdens (Yeo et al. 2015). As discussed by the authors, understanding the bioactivities of the drug-free and drug-incorporating carriers and their implications in

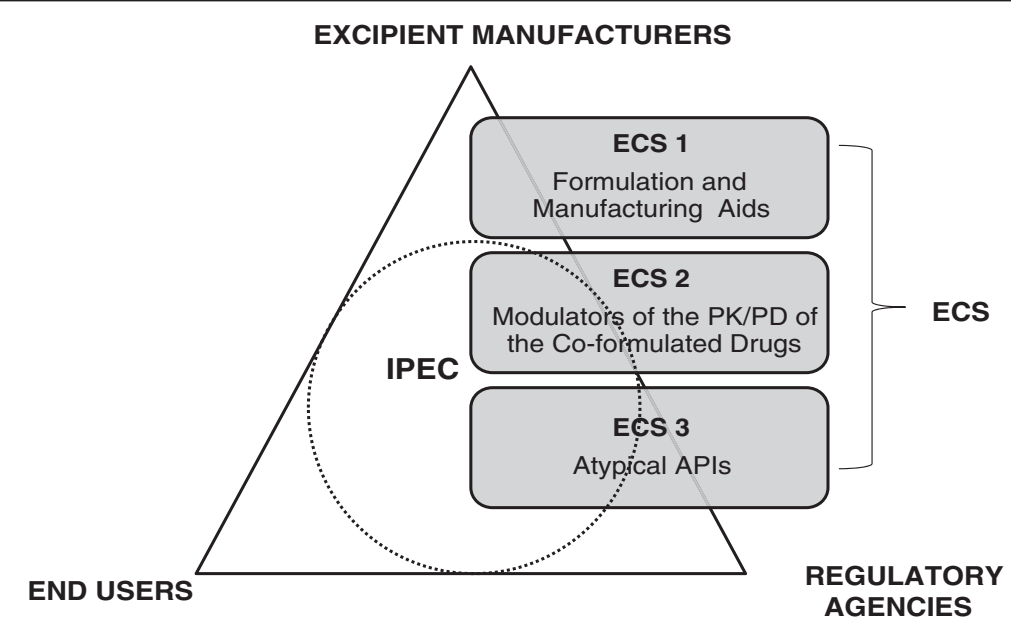

Fig. 2 Interplay between a proposed Excipient Classification System (ECS) and major stakeholders. IPEC designates International Pharmaceutical Excipients Council and API active pharmaceutical ingredient 
drug product development is very important to optimize drug product safety and performance.

\section{Recommendations}

Though the above facts are well known, a system to classify excipients on the basis of their in vivo functionality and ability to significantly influence the therapeutic activity of co-formulated drugs or their use as atypical active pharmaceutical ingredients is still unavailable. This is particularly important for novel excipients and new uses of approved excipients. Driving forces behind a new use of an approved excipient, include, addressing unmet medical need(s), no product without the excipient, patent protection and revenues. Understanding excipient effects (in vitro/in vivo) and development timeline constraints including toxicity and safety requirements are critical to the new use of an approved excipient as well to the use of a novel excipient. The International Pharmaceutical Excipients Council (IPEC) has been a strong advocate of the qualification and uses of excipients and authored many publications and meeting proceedings that deal with excipient functionality, specifications, characterization, manufacturing and quality-by-design $(\mathrm{QbD})$ aspects as well as toxicity and safety aspects especially with novel excipients (DeMerlis et al. 2009; Moreton 2010; IPEC Americas website http://www.ipecamericas.org). A classification which currently exists as indicated in the Handbook of Pharmaceutical Excipients (Rowe et al. 2009) depends on their role in the dosage form and manufacturing application like lubricants, glidants, emulsifiers, fillers, rate controlling agents, preservatives etc. However, this classification system (Rowe et al. 2009) is based on the function of the excipient in the formulation and dosage form and fails to address non-traditional uses of biologically "active" excipients as presented in this commentary. Therefore, it is recommended that a broad 'Excipient Classification System' (ECS) be considered and introduced to segregate the excipients used as formulation and manufacturing aids from those affecting the absorption, distribution, metabolism, and elimination (ADME) and PK/PD properties of the co-administered drugs. This would help both established and new researchers in the area to clearly understand the role of excipients, assist in their judicious selection and interpretation of the effect of formulation variables on the in vivo performance of the dosage forms. Furthermore, ECS would be instrumental for the regulatory bodies to set standards and introduce appropriate guidelines to control within required limits or allow minimum variation in use of such excipients in post approval cases and also recommend submitting of adequate information/data related to their use in new drug products. It should also facilitate the approval process for novel excipients. A suggested system may include for example, inactive/inert excipients used as formulation and manufacturing aids versus excipients exhibiting biological activity, where the latter can be further subdivided in excipients that modulate the PK/PD of the co-formulated drug versus excipients which are atypical active pharmaceutical ingredients. Properties such as membrane permeability modulation, gut wall metabolism and efflux pump inhibition can be considered for the excipients that affect the PK/PD of the co-formulated drug.

Input from all stakeholders, that is, the excipient manufacturers, end users in the biotech and pharmaceutical industry, the International Pharmaceutical Excipients Council (IPEC) and regulatory agencies such as the Inactive Ingredient Subcommittee (IIS) of the CDER of the FDA, is needed before a widely accepted ECS is implemented. Figure 2 illustrates the interplay between these stakeholders along with the proposed tentative three classes of excipients for oral drugs.

\section{Conclusion}

It is hoped that this commentary on excipients and ECS recommendation, would stimulate constructive discussion on this important issue of pharmaceutical excipients before an appropriate ECS becomes a reality. While this commentary is focused on oral drug products, discussed excipient considerations and recommendations can be broadly applied to excipients used in other routes of administration.

\section{Abbreviations}

ADME: absorption, distribution, metabolism, elimination; API: active pharmaceutical ingredient; BCS: biopharmaceutics classification scheme; CDER: center for drug evaluation and research; ECS: Excipient Classification System; FDA: food and drug administration; IIS: Inactive Ingredient Subcommittee; IPEC: International Pharmaceutical Excipients Council; OTC: over-the-counter; PEG: polyethylene glycol; PD: Pharmacodynamics; P-gp: P-glycoprotein; PK: pharmacokinetics; TPGS: a-tocopherol-polyethylene glycol 1000-succinate; QbD: quality-by-design; UDP: uridinyl disphosphate.

\section{Author details}

${ }^{1}$ Biopharmaceutical \& Drug Delivery Consulting, LLC, P. O. Box 7565, Gurnee, IL 60031-6226, USA. ${ }^{2}$ Evonik India Pvt, Ltd, Mumbai, India. ${ }^{3}$ Department of Pharmaceutics, Indian Institute of Technology (BHU), Varanasi, India.

Received: 16 February 2016 Accepted: 28 March 2016

Published online: 10 May 2016

\footnotetext{
References

Alasino RV, Ausar SF, Bianco ID, Castagna LF, Contigiani M, Beltramo DM (2005) Amphipathic and membrane-destabilizing properties of the cationic acrylate polymer Eudragit ${ }^{\oplus}$ E100. Macromol Biosci 5:207-213

Aungst BJ (2012) Absorption enhancers: applications and advances. AAPS J 14:10-17

Boddu, Sai HS, Renukuntla J, Rega, A, Alexander K. (2015) Excipients and non-medicinal agents as active pharmaceutical ingredients. In Narang AS, Boddu, Sai HS eds Excipient Applications in Formulation Design and Drug Delivery. Springer, 2015 pp 613-636.

Chen ML (2008) Lipid Excipients and delivery systems for pharmaceutical development: a regulatory perspective. Adv Drug Del Rev 60:768-777 Chen M-L, Sadrich N, Yu L (2013) Impact of Osmotically Active Excipients on Bioavailability and Bioequivalence of BCS III Drugs. AAPSJ 15:1043-1050 Chakraborty S, Shukla D, Mishra B, Singh S (2009) Lipid - An emerging platform for oral delivery of drugs with poor bioavailability. Eur J Pharm Biopharm 73:1-15
} 
Constantinides PP, Han J, Davis SS (2006) Advances in the use of tocols as drug delivery vehicles. Pharm Res 23:243-255

Constantinides PP, Wasan KM (2007) Lipid formulation strategies for enhancing intestinal transport and absorption of P-glycoprotein (P-gp) substrate drugs: In vitro/In vivo case studies. J Pharm Sci 96:235-248

Constantinides PP (2014) Excipients as Inhibitors of the Absorption of Dietary Cholesterol: Principles and Case Studies, symposium on Excipients as Atypical Actives in Nutraceuticals and Pharmaceuticals: Applications and Development Considerations. AAPS Annual Meeting, San Diego, CA

DeMerlis C, Goldring J, Velagaleti R, Brock W, Osterberg R. (2009). Regulatory Update: The IPEC Novel Excipient Safety Evaluation Procedure. Pharm Tech 33 (11)

Drakulich A (2011) Atypical actives gain attention: clarifying GMPs for excipients used as actives. Pharm Technol 35:s41-s42

George M, Abraham TE (2006) Polyionic hydrocolloids for the intestinal delivery of protein drugs: Alginate and Chitosan-a review. J Control Rel 114:1-14

Gershkovich P, Darlington J, Constantinides PP, Wasan KM (2009) Inhibition of intestinal absorption of cholesterol by surface-modified nanostructured aluminosilicate (NSAS) compounds. J Pharm Sci 98:2390-2400

Grube S, Wolfrum U, Langguth P (2008) Characterization of the epithelial permeation enhancing effect of basic butylated methacrylate copolymer in vitro studies. Biomacromol 9:1398-1405

Haydel SE, Remenih CM, Williams LB (2008) Broad-spectrum in vitro antibacterial activities of clay minerals against antibiotic-susceptible and antibiotic-resistant bacterial pathogens. J Antimicrob Chemother 61:353-361

IPEC Americas Website : http://www.ipecamericas.org

Maher S, Leonard TW, Jacobsen J, Brayden DJ (2009) Safety and efficacy of sodium caprate in promoting oral drug absorption: from in vitro to the clinic. Adv Drug Del Rev 61:1427-1449

Moreton RC (2010) Trends in Pharmaceutical Excipients: An Update. Am Pharm Rev 13:22-27

Qi S, Gryczke A, Graig DQ (2008) Characterization of solid dispersions of paracetamol and EUDRAGIT E prepared by hot-melt extrusion using thermal, microthermal and spectroscopic analysis. Int J Pharm 354:158-167

Rowe CR, Sheskey PJ, Quinn ME (2009) Handbook of Pharmaceutical Excipients, 6th edn. American Pharmacists Association, Pharmaceutical Press, Washington, DC

Shukla D, Chakraborty S, Singh S, Mishra B (2011) Lipid based oral multiparticulate formulations - Advantages, technological advances and industrial applications. Expert Opin Drug Deliv 8:207-224

Six K, Verreck G, Peeters J, Brewster M, Van Der Mooter G (2004) Increased physical stability and improved dissolution properties of itraconazole, a class II drug, by solid dispersions that combine fast- and slow-dissolving polymers. J Pharm Sci 93:124-131

Strickley RG (2004) Solubilizing excipients in oral and injectable applications. Pharm Res 21:201-230

Wang H-J, Hsiong C-H, Ho S-T, Lin M-J, Shih T-Y, Huang P-W, Hu OY-P (2014) Commonly Used Excipients Modulate UDP-Glucuronosyltransferase 2B7 Activity to Improve Nalbuphine Oral Bioavailability in Humans. Pharm Res 31:1676-1688

Wasan KM (ed) (2007) Role of Lipid Excipients in Modifying Oral and Parenteral Drug Delivery: Basic Principles and Biological Examples. Wiley, New York

Whitehead K, Karr N, Mitragotri S (2008) Safe and effective permeation enhancers for oral drug delivery. Pharm Res 25:1782-1788

Yeo Y, Kim B-K (2015) Drug Carriers: Not an Innocent Delivery Man. AAPS J 17:1096-1103

\section{Submit your manuscript to a SpringerOpen ${ }^{\circ}$ journal and benefit from:}

- Convenient online submission

- Rigorous peer review

- Immediate publication on acceptance

- Open access: articles freely available online

- High visibility within the field

Retaining the copyright to your article

Submit your next manuscript at $\boldsymbol{s p r i n g e r o p e n . c o m ~}$ 